\section{Zahlen des Monats}

\section{Fokus auf Prävention}

Für die Zahnärzte stehen Prävention und Prophylaxe schon lange im Fokus. Inzwischen ist das Thema Vorsorge auch bei den gesetzlichen Krankenkassen groß im Rennen. Laut aktuellem Präventionsbericht, der vom GKV-Spitzenverband und dem Medizinischem Dienst des GKV-Spitzenverbandes herausgegeben wird, haben die gesetzlichen Krankenkassen 2014 insgesamt 293 Millionen Euro für Gesundheitsförderung und Primärprävention ausgegeben. Das waren zehn Prozent mehr als im Jahr davor. Besonders viel haben die Kassen mit knapp 68 Millionen Euro in die betriebliche Gesundheitsförderung investiert. Von großer Bedeutung sei auch Prävention in Kindergärten, Schulen, Wohngebieten und Stadteilen, heißt es in dem Bericht.

\section{Boom auf Arbeitsmarkt}

Gesunde Menschen sind auf dem Arbeitsmarkt besonders gefragt. Und dieser Markt floriert derzeit. Die Zahl der Arbeitslosen in Deutschland war im November 2015 so gering wie seit 24 Jahren nicht mehr. Wie die Bundesagentur für Arbeit mitteilte, ist die Arbeitslosenzahl im vergangenen November um 16.000 Menschen auf 2,633 Millionen gesunken. Weniger Jobsuchende gab es zuletzt 1991. Nach Angaben des Statistischen Bundesamtes gab es im Oktober 2015 insgesamt 43,4 Millionen Erwerbstätige. Das ist der höchste Stand seit der Wiedervereinigung.

\section{Run auf Hochschulen}

Und noch ein Rekord: Im Wintersemester 2015/2016 sind so viele Studenten wie noch nie an deutschen Hochschulen immatrikuliert. Laut Statistischem Bundesamt studieren nach ersten vorläufigen Ergebnissen rund 2.8 Millionen Frauen und Männer. Damit erhöhte sich die Zahl der Studierenden im Vergleich zum Wintersemester 2014/2015 um gut 60.000 oder 2,2 Prozent. Unter den Bundesländern am meisten Zuwachs hatte Niedersachsen mit 5,4 Prozent mehr Studierende als im Wintersemester zuvor.

Ohne große Diskussion im Bundestag

\title{
Pünktlich zum Jahreswechsel: E-Health-Gesetz ist in Kraft getreten
}

Gegen die Stimmen der Links-Fraktion und bei Enthaltung der Grünen hat der Deutsche Bundestag das „Gesetz für sichere digitale Kommunikation und Anwendungen im Gesundheitswesen " kurz E-Health-Gesetz - im Dezember verabschiedet, damit es pünktlich zum Jahreswechsel in Kraft treten konnte. Das neue Gesetz soll die Grundlage für die Einführung weiterer Funktionen der elektronischen Gesundheitskarte, den Aufbau einer Telematikinfrastruktur für eine sichere Kommunikation im Gesundheitswesen und die Förderung telemedizinischer Anwendungen sein.

Die elektronische Prüfung und Aktualisierung von Stammdaten der Versicherten soll binnen zwei Jahren flächendeckend in Deutschland eingeführt werden. Mit dem E-Health-Gesetz wird auch der Einstieg in die elektronische Patientenakte gefördert: Die Gesellschaft für Telematikanwendungen (gematik) muss bis Ende 2018 die Voraussetzungen dafür schaffen, dass Daten der Patienten einer elektronischen Patientenakte für die $\mathrm{Pa}$ tienten bereitgestellt werden können. $\mathrm{Ab}$ 1. Juli 2018 sind pauschale Kürzungen der Vergütung der Ärzte und Zahnärzte vorgesehen, die nicht an der Online-Prüfung der Versichertenstammdaten teilneh- men. Von 2018 an sollen Daten auf Wunsch der Patienten zu Allergien oder Vorerkrankungen, so genannte Notfalldaten, auf der elektronischen Gesundheitskarte gespeichert werden können. Außerdem sollen Medikationspläne erstellt werden können. Personen, die mindestens drei Arzneimittel gleichzeitig verschrieben bekommen, haben ab dann Anspruch auf einen genauen Plan zur Einnahme. Zudem sollen ab Mitte 2018 die auf der Karte gespeicherten Daten in der Arztpraxis auch mit den Krankenkassen abgeglichen werden können und sich aktualisieren lassen, der Medikationsplan kann dann ab 2019 zudem in der Apotheke aktualisiert werden, sofern der Versicherte zuvor dem Datenabgleich zugestimmt hat.

Laut einer Berfragung des InternetVerbandes Bitkom steht eine große Mehrheit der Deutschen zusätzlichen Funktionen für die elektronische Gesundheitskarte aufgeschlossen gegenüber. Für Bitkom-Hauptgeschäftsführer Bernhard Rohleder überwiegen die Vorteile der neuen Technik: „Die Speicherung entsprechender Informationen auf der elektronischen Gesundheitskarte macht das Leben für Patienten und Ärzte nicht nur leichter, sie wird Leben retten." $\quad p k /$ red

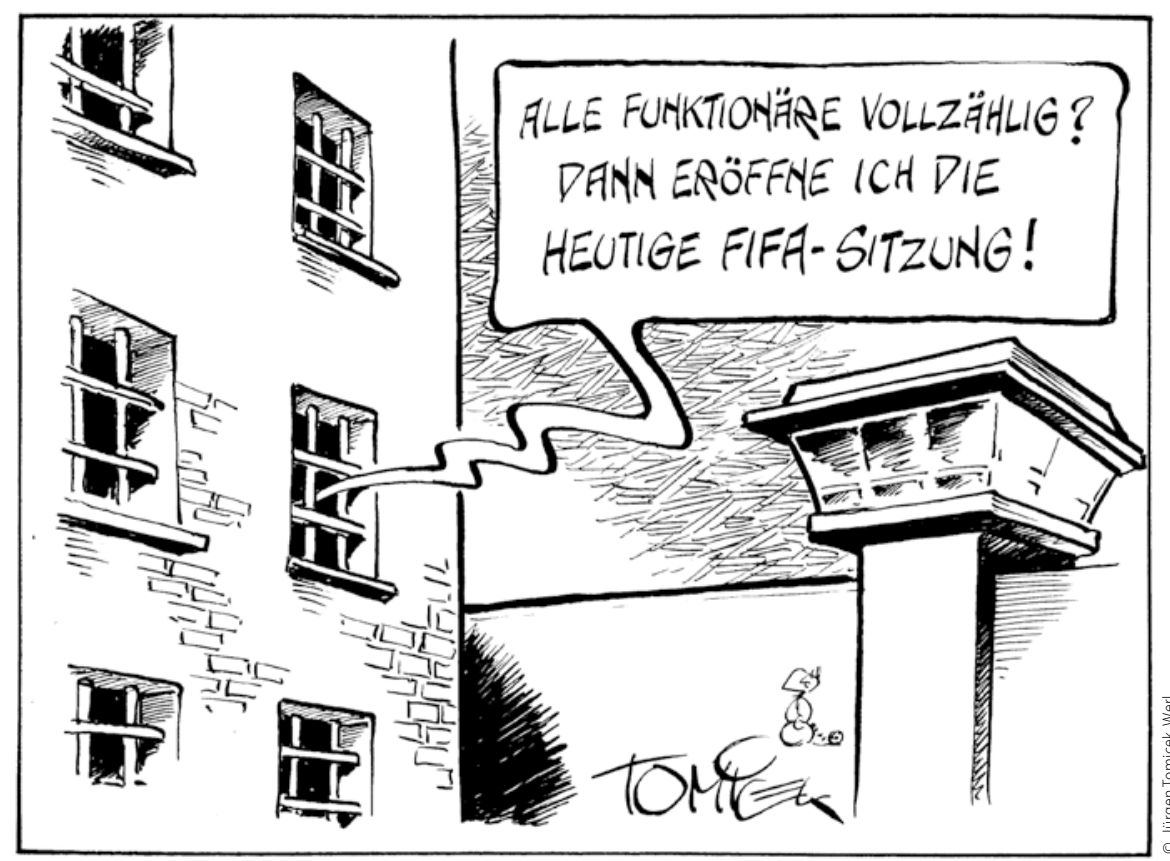

